\title{
On using peak amplitude and rise time for AE source characterization
}

\author{
M A MAJEED* and C R L MURTHY \\ Department of Aerospace Engineering, Indian Institute of Science, \\ Bangalore 560 012, India \\ *Present address: Department of Computer Science \& Engineering, Noorul Islam \\ College of Engineering, Kumaracoil, Thuckalay, India \\ e-mail: ponnara@mailcity.com; crlmurty@aero.iisci.ernet.in
}

MS received 13 June 2001; revised 18 December 2001

\begin{abstract}
Acoustic Emission (AE) signals, which are electrical version of acoustic emissions, are usually analysed using a set of signal parameters. The major objective of signal analysis is to study the characteristics of the sources of emissions. Peak amplitude $\left(P_{a}\right)$ and rise time $\left(R_{t}\right)$ are two such parameters used for source characterization. In this paper, we theoretically investigate the efficiency of $P_{a}$ and $R_{t}$ to classify and characterize $\mathrm{AE}$ sources by modelling the input stress pulse and transducer. Analytical expressions obtained for $P_{a}$ and $R_{t}$ clearly indicate their use and efficiency for source characterization. It is believed that these results may be of use to investigators in areas like control systems and signal processing also.
\end{abstract}

Keywords. Acoustic emission; source characterization; efficient parameters; peak amplitude; rise time.

\section{Introduction}

Acoustic Emissions (AE) are stress waves produced in materials by irreversible deformation processes like crack growth. AE signals are the electrical form of acoustic emissions containing vital information about the sources (i.e. deformation processes) that produce the emissions. When AE is used as a non-destructive evaluation tool, this information is extracted using a set of parameters. $P_{a}$ and $R_{t}$ are two such significant parameters which have been used in several investigations reported in the literature. (e.g. Bhat \& Murthy 1993, Murthy et al 1985, 1987). The success of many of these investigations point to the utility of $P_{a}$ and $R_{t}$ for source characterization. However, these investigations are more or less feasibility studies, and it cannot be concluded from these studies that $P_{a}$ and $R_{t}$ are useful for all situations of signal analysis, carried out for source characterization because there is no proper theoretical basis for their use in these investigations. More systematic investigations are required before using these parameters in a wider class of signal-analysis problems. The efficiency of these parameters, we believe, has to be established in a general theoretical frame work. This belief has motivated us to carry out the investigations reported in the following. 


\section{The approach}

Our approach to studying $P_{a}$ and $R_{t}$, from source characterization point of view, consists in mathematically modelling the transient stress pulse and the transducer which converts it to an electrical signal . This model is used to obtain analytical expressions for $P_{a}$ and $R_{t}$ which are then used to study these parameters from source characterization point of view.

\subsection{Stress pulse}

So far it has not been possible to record an emission event or stress pulse as it leaves the source. Neither is the physics of the wide variety of deformation mechanisms that give rise to AE fully understood. Hence, modelling stress pulses exactly appears to be rather difficult. However, this should not deter us from pursuing the stated goal of theoretically studying the efficiency of $P_{a}$ and $R_{t}$, as stress pulses can reasonably be assumed to be of the form of typical transient functions fully mathematically defined in the literature. The functions used in our investigation are listed below along with their Fourier transforms, which are available in the signal processing literature.

(e.g. Salivahanan et al 2000)

(i) Triangular functions (figure 1):

$$
i_{T}(t)= \begin{cases}A(1+2 t / T), & -T / 2 \leq t \leq 0 \\ A(1-2 t / T), & 0=t=T / 2 \\ 0, \text { otherwise. } & \end{cases}
$$

In the following the maximum amplitude $A$ of the triangular function is referred to as its pulse height.

Fourier transform:

$$
I_{T}(f)=(A T / 2) \frac{\sin ^{2}(\Pi T / 2)}{(\Pi f T / 2)^{2}} .
$$

(ii) Gaussian function (figure 2):

$$
i_{G}(t)=A \exp \left(-t^{2} / 2 \sigma^{2}\right) .
$$

Fourier transform :

$$
I G(f)=(2 \Pi)^{1 / 2} A \sigma \exp \left[-\sigma^{2} 4 \Pi^{2} f^{2} / 2\right] .
$$

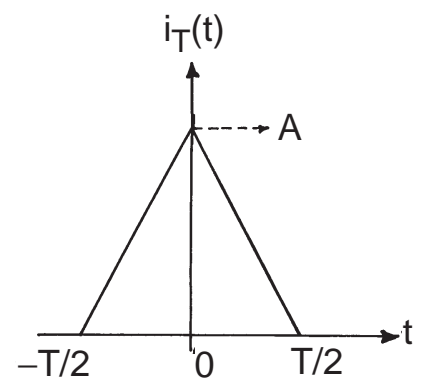

Figure 1. Triangular function. 


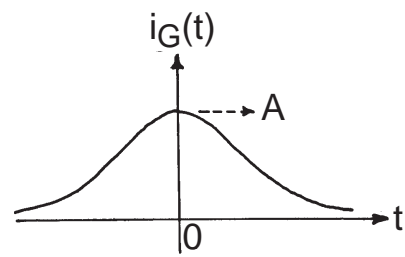

Figure 2. Gaussian function.

More than $99.87 \%$ of the total area enclosed by the Gaussian curve of (3) lies between $t=-3 \sigma$ and $t=3 \sigma$. Hence, for analytical purpose, (3) can be redefined as a finiteduration function.

$$
i_{G}(t)=\left\{\begin{array}{l}
A \exp \left(-t^{2} / 2 s^{2}\right), \quad-3 \sigma=t=3 \sigma, \\
0, \text { otherwise. }
\end{array}\right.
$$

Thus the pulse width of the Gaussian function (truncated) $T=6 \sigma$. In the sequel, by pulse height of the Gaussian function we mean its maximum amplitude $A$.

(iii) Rectangular function (figure 3)

$$
i_{R}(t)= \begin{cases}A, & -T / 2 \leq t \leq T / 2, \\ 0, \text { otherwise. } & \end{cases}
$$

Fourier transform:

$$
I_{R}(f)=A T[(\sin \Pi f T) / \Pi f T]
$$

(iv) Half cosine function (figure 4)

$$
i_{C}(t)=\left\{\begin{array}{l}
A \cos (\Pi t / T), \quad-T / 2 \leq t \leq T / 2, \\
0, \text { otherwise. }
\end{array}\right.
$$

In the sequel, the maximum amplitude $A$ is referred to as the height of the half cosine pulse.

Fourier transform:

$$
I_{C}(f)=[(2 A \Pi) /(T)]\left[(\cos \Pi f T) /\left((\Pi / T)^{2}-(2 \Pi f)^{2}\right)\right] .
$$

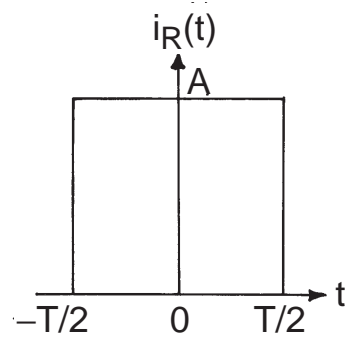

Figure 3. Rectangular function. 


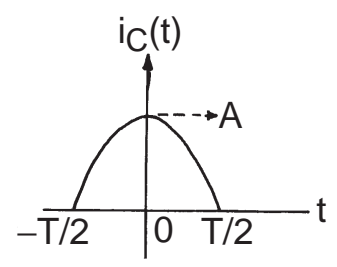

Figure 4. Half cosine function.

\subsection{Basis for the choice}

Our choice of rectangular and triangular functions is based on the physics of the phenomenon presented by Hill \& Stephens (1974). In this model, acoustic emission is assumed to be generated by the displacment of any defect. Three distinct phases, viz., (1) initial phase of constant acceleration, (2) intermediate phase of steady growth - with constant velocity, and (3) final phase of constant deceleration, are identified in the total course of the displacement of the defect. The stress waveform derived from this defect-displacement model has the form shown in figure 5 .

A close look at the waveform suggests that if the initial and final phases are of negligible duration, then the stress waveform (figure 5) can be approximated by a rectangular function. In situations where the intermediate phase is of negligible duration compared to the other two phases, the stress waveform can be approximated by a triangular function.

Choice of the truncated Gaussian function, (5) and half cosine function can be justified in the light of the work by Stephens \& Pollock (1971). In this, by considering the fundamental constraints that the stress wave emitted from irreversible plastic deformation of the test specimen should satisfy, the authors propose a pulse of the form shown in figure 6 as a stress waveform model. Two functions that resemble this model in shape are the truncated Gaussian function and the half cosine function.

\subsection{Transducer modelling}

For ease of analysis, it is assumed that the transducer is a linear time-invariant system (Papoulis 1977) with ideal bandpass characteristics. Hence, frequency response $H(f)$ of the transducer

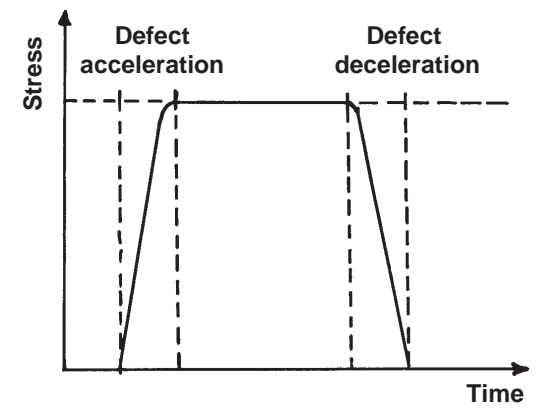

Figure 5. Theoretical stress waveform from Hill \& Stephens (1974). 


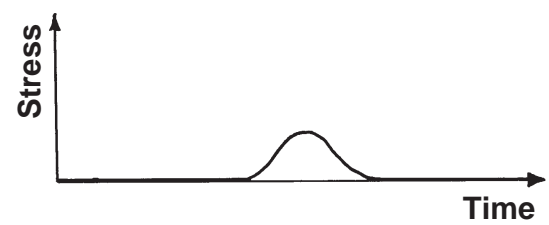

Figure 6. Theoretical stress waveform from Stephens \& Pollock (1971).

can be written as

$$
H(f)=\left\{\begin{array}{l}
C \exp \left(-j 2 \Pi f t_{0}\right), \quad f_{1}<|f|<f_{2}, \\
0, \text { otherwise }
\end{array}\right.
$$

where, $t_{0}$ is the transmission time or delay constant (Papoulis 1977) of the transducer and $C$, a constant expressed in volts per microbar $\left(10^{-1} \mathrm{~Pa}\right)$.

\subsection{Transducer response to transient functions}

The stress wave emitted by the source propagates through the material and is distorted when it reaches the transducer. However, for analytical simplicity, it is assumed that the transducer is close to the source and that the stress wave reaches the transducer just as it leaves the source. Under this assumption and the assumption made in the previous section, the Fourier transform $G(f)$ of the signal $g(t)$ due to an input stress pulse $i(t)$ is given by

$$
G(f)=I(f) H(f),
$$

where $I(f)$ is the Fourier transform of $i(t)$. Hence,

$$
g(t)=\int_{-00}^{+00} I(f) H(f) \exp (j 2 \Pi f t) \mathrm{d} f .
$$

Substituting for $H(f)$ from (10), we get

$$
g(t)=C \int_{-f 2}^{-f 1} I(f) \exp \left[j 2 \Pi f\left(t-t_{0}\right)\right] \mathrm{d} f+C \int_{f 1}^{f 2} I(f) \exp \left[j 2 \Pi f\left(t-t_{0}\right)\right] \mathrm{d} f,
$$

which reduces to (Papoulis 1977),

$$
g(t)=2 C \int_{f 1}^{f 2} I(f) \cos 2 \Pi f\left(t-t_{0}\right) \mathrm{d} f .
$$

Equation (13) can be used by substituting for $I(f)$, to find the transducer response to the input stress pulses described in $\$ 2.1$.

Triangular input: The output due to the triangular input defined by (1) is given by

$$
g_{T}(t)=C A T \int_{f 1}^{f 2} \frac{\sin ^{2}(\Pi f T / 2)}{(\Pi f T / 2)^{2}} \cos 2 \Pi f\left(t-t_{0}\right) \mathrm{d} f .
$$




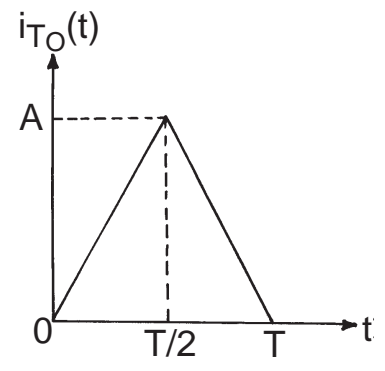

Figure 7. Delayed triangular function.

This represents the transducer response to a triangular stress pulse that originates at time $t=-T / 2$. However, for real-time measurements of signal parameters, it is more appropriate to consider input functions that are applied to the transducer input at non-negative instances of time. For analytical convenience, we take the origin of the time axis as the instant when the stress pulse arrives at the transducer input. Thus, if the input is triangular, we have to find the transducer response to an input pulse of the form shown in figure 7. It is not difficult to see that this is same as the function shown in figure 1 and defined in (1), but shifted in time by a factor $T / 2$. If $i_{T o}(t)$ denotes this function, then

$$
i_{T o}(t)=i_{T}(t-T / 2) \text {. }
$$

Therefore, the output due to this stress pulse, assuming the time-invariance (Papoulis 1977) property of the transducer is given by

$$
g_{T o}(t)=g_{T}(t-T / 2)
$$

i.e.

$$
g_{T o}(t)=C A T \int_{f 1}^{f 2} \frac{\sin ^{2}(\Pi f T / 2)}{(\Pi f T / 2)^{2}} \cos 2 \Pi f\left[t-\left(t_{0}+T / 2\right)\right] \mathrm{d} f .
$$

Using the notation $\mathrm{gT}(\mathrm{t})$ to denote $\mathrm{gTo}(\mathrm{t}),(15)$ may be rewritten as

$$
g_{T}(t)=C A T \int_{f 1}^{f 2} \frac{\sin ^{2}(\Pi f T / 2)}{(\Pi f T / 2)^{2}} \cos 2 \Pi f\left[t-\left(t_{0}+T / 2\right)\right] \mathrm{d} f .
$$

Gaussian input: The function shown in figure 8 can be written in terms of the function defined in (5) as

$$
i_{G o}(t)=i_{G}(t-3 \sigma) .
$$

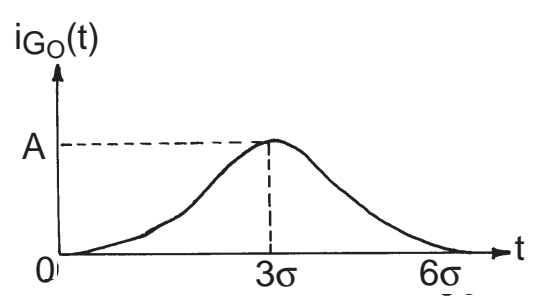

Figure 8. Delayed Gaussian function. 


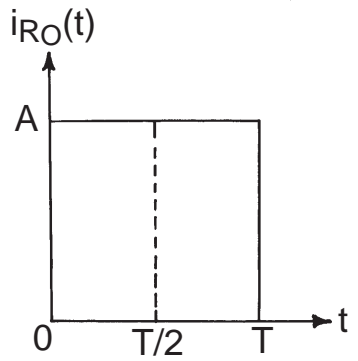

Figure 9. Delayed rectangular function.

If this stress pulse is applied to the transducer input, the output $g_{G}(t)$, by the same line of argument as for the triangular input, is given by

$$
g G(t)=2(2 \Pi)^{1 / 2} C A \sigma \int_{f 1}^{f 2} \exp \left(-\sigma^{2} 4 \Pi^{2} f^{2} / 2\right) \cos 2 \Pi f\left[t-\left(t_{0}+3 \sigma\right)\right] \mathrm{d} f .
$$

Rectangular input: The output signal $\mathrm{gR}(\mathrm{t})$ due to the delayed rectangular input $i_{R o}(t)$ shown in figure 9 can be written as the output signal due to the rectangular input of figure 3 delayed in time by a factor $\mathrm{T} / 2$. That is,

$$
g_{R}(t)=2 C A T \int_{f 1}^{f 2} \frac{\sin \Pi f T}{\Pi f T} \cos 2 \Pi f\left[t-\left(t_{0}+T / 2\right)\right] \mathrm{d} f .
$$

Half cosine input: The output signal $g_{C}(t)$ due to the delayed half cosine input (figure 10) can be written as

$$
g_{C}(t)=\frac{4 C A \Pi}{T} \int_{f 1}^{f 2} \frac{\cos \Pi f T}{(\Pi / T)^{2}-(2 \Pi f)^{2}} \cos 2 \Pi f\left[t-\left(t_{0}+T / 2\right)\right] \mathrm{d} f .
$$

\section{Theoretical peak amplitude and rise time}

Peak amplitude $\left(P_{a}\right)$ and rise time $\left(R_{t}\right)$ have been conventionally used in several AE applications, though there is no proper theoretical basis for their use, as stated in the introduction. Hence, it is worthwhile to study the significance of $P_{a}$ and $R_{t}$ as parameters for source characterisation using the transducer model described in $\S 2.3$. We derive theoretical expressions for $P_{a}$ and $R_{t}$ and study the efficiency of these parameters for source characterization in the following.

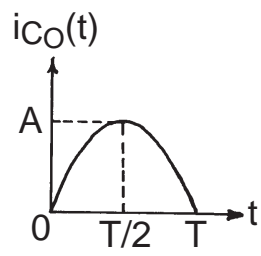

Figure 10. Delayed half cosine function. 


\section{1 $P_{a}$ and $R_{t}$ for triangular input function}

Suppose that sources are triangular stress pulses which originate and arrive at the transducer input at time $t=0$. The output due to such a stress pulse is given by (16) from which we get the peak amplitude $P_{a}$ (i.e. $\max g_{T}(t)$ ) as

$$
P_{a}=C A T \int_{f 1}^{f 2} \frac{\sin ^{2}(\Pi f T / 2)}{(\Pi f T / 2)^{2}} \mathrm{~d} f .
$$

Similarly, the rise time $R_{t}$ is given by

$$
R_{t}=T / 2 .
$$

Equations (20) and (21) are proved in appendix A.

\section{$3.2 P_{a}$ and $R_{t}$ for gaussian input function}

The output signal for Gaussian stress pulses (figure 7) is given by (17). $P_{a}$ of this signal is given by

$$
\left.P_{a}=\max g_{G}(t)=2 \sqrt{(} 2 \Pi\right) C A \sigma \int_{f 1}^{f 2} \exp \left[-\sigma^{2} 4 \Pi^{2} f^{2} / 2\right] \mathrm{d} f .
$$

Equation (22) can be proved along the same lines as (20). $P_{a}$ occurs uniquely at $t=t_{0}+3 \sigma$. Uniqueness of $P_{a}$ can also be proved by the same line of arguments as for the triangular function.

If we take, as in the case of the triangular input function, time origin as the instant when the signal first appears at the transducer output, i.e., treating $t_{0}=0$, we get $R_{t}$ measured from the origin as

$$
R_{t}=3 \sigma
$$

Since the total duration or pulse width of the Gaussian pulse (figure 7) is $T=6 \sigma$. Hence the equation $R_{t}=3 \sigma$ states that $R_{t}$ as measured from the beginning of the signal is equal to half the pulse width $T$. That is,

$$
R_{t}=T / 2 .
$$

This relationship between $R_{t}$ and $T$ is the same as that between them in the case of the triangular input, (21).

\section{3 $P_{a}$ and $R_{t}$ for rectangular and half cosine input functions}

The output signal due to the rectangular input of figure 9 is given by (18). If the pulse width $T$ and the upper cut-off frequency $f_{2}$ are such that $f_{2} T \leq 1$, i.e. $\Pi f_{2} T \leq \Pi$ for all $f, f_{1} \leq f \leq f_{2}$, then $\sin \Pi f t / \Pi f t \geq 0$. Under this condition a closed-form expression for $P_{a}$ and $R_{t}$ analogous to those for triangular and Gaussian input functions can be obtained as

$$
P_{a}=\max g_{R}(t)=2 C A T \int_{f 1}^{f 2} \frac{\sin \Pi f T}{\Pi f t} \mathrm{~d} f
$$


and

$$
R t=T / 2 .
$$

The output signal for half cosine input function of figure 10 is given by (19) and the function $\cos \Pi f T /\left[(\Pi / T)^{2}-(2 \Pi f)^{2}\right]$ takes the positive maximum value when $\Pi f T=0$, and crosses zero first when $\Pi f T=3 \Pi / 2$.

Suppose that $T$ and $f_{2}$ are such that

$$
\Pi f_{2} T \leq 3 \Pi / 2 \text {, i.e. } f_{2} \leq 3 / 2 T \text { or } T \leq 3 / 2 f_{2} .
$$

Under this condition $\cos \Pi f T /\left[(\Pi / T)^{2}-(2 \Pi f)^{2}\right] \geq 0$ for all $f, f_{1} \leq f \leq f_{2}$ and hence $P_{a}$ is given by

$$
P_{a}=\frac{4 C A \Pi}{T} \int_{f 1}^{f 2} \cos \Pi f T /(\Pi / T)^{2}-(2 \Pi f)^{2} \mathrm{~d} f,
$$

and

$$
R_{t}=T / 2
$$

In (25) and (27), it is assumed that the time origin is the instant when the signal first appears at the transducer output.

\section{Verification using simulated signals}

Results given by (20), (22), (24) and (26) and the relation $R_{t}=T / 2$ do not exist, to the best knowledge of the present authors, in signal processing, control system, or any other related technical literature. In order to ascertain the validity of these equations, we simulated signals by numerically integrating (16)-(19). The procedure adopted is the Gauss-Legendre quadrature formula which is one of the most accurate numerical integration procedures. Gauss-Legendre approximation for the integral of any function $f(x)$ over the interval $(-1,1)$ is given by (Krylov 1966),

$$
\int_{-1}^{1} f(x) \mathrm{d} x \approx \sum_{i=1}^{N} w_{i} f\left(\lambda_{i}\right)
$$

where $N$ is the number of points or nodes, $\left(\lambda_{i}\right)$ the zeros of the Legendre polynomial of degree $N$, and $\left[w_{i}\right]$ the appropriate weight factors. $\int_{a}^{b} f(x) \mathrm{d} x$ can be obtained from $\int_{-1}^{1} f(x) \mathrm{d} x$ by the transformation i.e.

$$
\int_{a}^{b} f(x) \mathrm{d} x=\frac{b-a}{2} \int_{-1}^{1} f\left[\frac{b-a}{2} y+\frac{a+b}{2}\right] \mathrm{d} y .
$$

The values $f_{1}=250 \mathrm{kHz}$ and $f_{2}=500 \mathrm{kHz}$ were mainly used to simulate the signals using (16)-(19), the integrals were evaluated at equidistant points of 0.5 microseconds for a maximum value of 100 microseconds. Transducer parameters defined in (10) were chosen as $f_{1}=250 \mathrm{kHz}, f_{2}=500 \mathrm{kHz}, C=1$ volt per microbar and $t_{0}=0$. The number of nodes $N=512$ was found to be adequate because as $N$ is increased from 48 to 512 , the resulting 


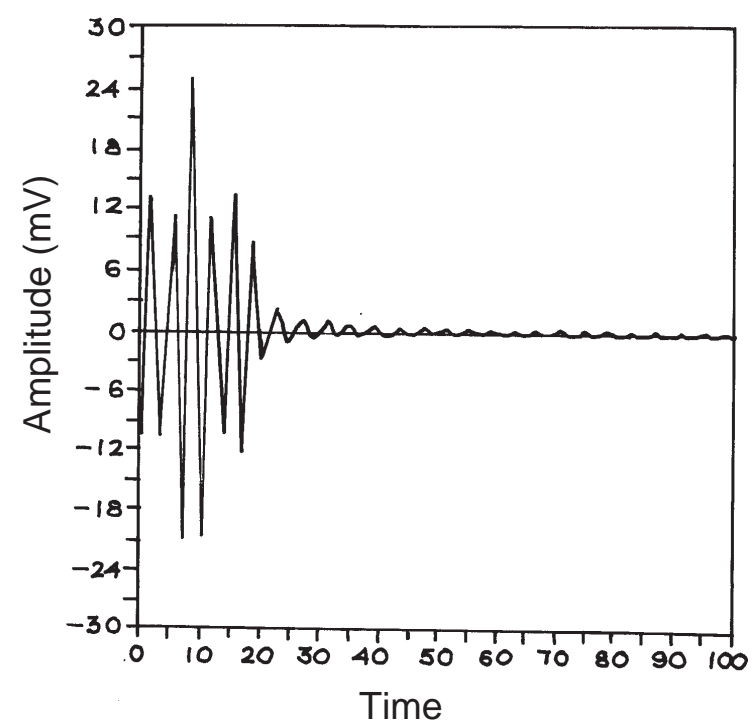

Figure 11. Signal due to a triangular source $T=17$ microseconds and $A=1$ microbar.

improvement in accuracy is not significant. $\left[\lambda_{i}\right]$ and $\left[w_{i}\right]$ were obtained as per Stroud \& Secrest (1966).

Large data bases of signals were simulated by using these four stress pulses, viz., triangular, Gaussian, rectangular and half cosine for three different pulse heights, viz., 1, 2, and 3 microbar and pulse widths varying from 1 to 30 microseconds. Four typical examples of these signals are shown in figurs 11-14.

Peak amplitude for triangular and Gaussian input pulses obtained by numerically integrating (20) and (22) were found to be the same as the peak amplitude of simulated signals, simulated using these input functions, there by proving the validity of these equa-

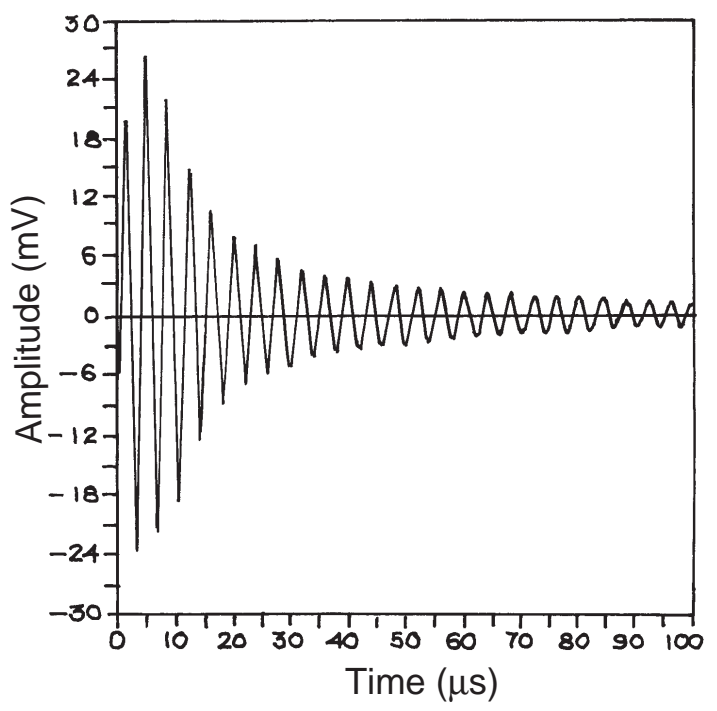

Figure 12. Signal due to a Gaussian source $T=10$ microseconds and $A=3$ microbar. 


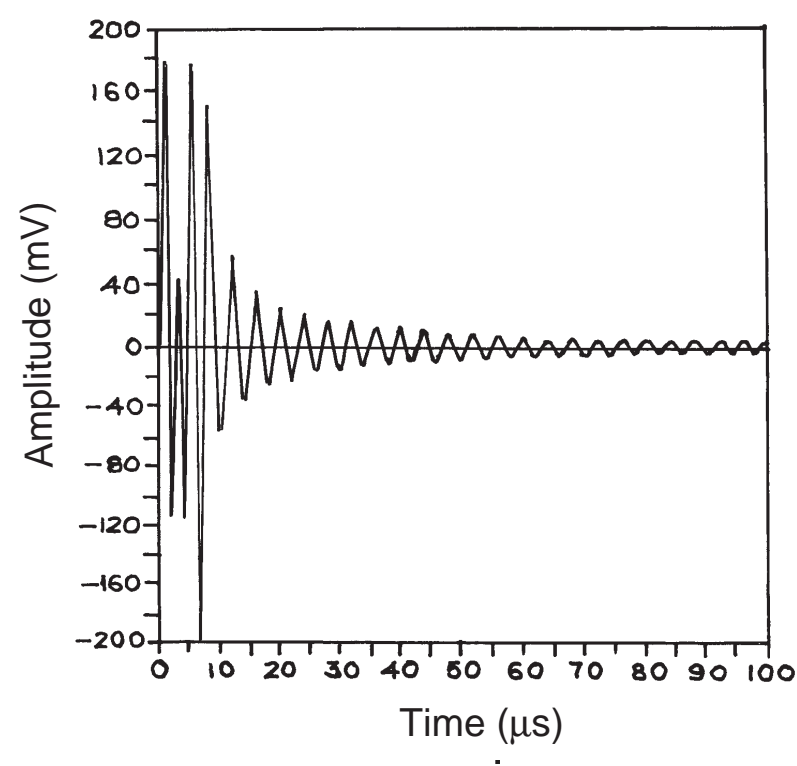

Figure 13. Signal due to rectangular source $T=5$ microseconds and $A=$ 3 microbar.

tions. Similarly the relation $R_{t}=T / 2$ was also found to be correct in the simulated signals.

Equations (24) and (25), for rectangular input, and (26) and (27), for half cosine input, have been derived under the conditions $f_{2} \leq 1 / T$ and $f_{2} \leq 3 / 2 T$ respectively. This means when $f 2=500 \mathrm{kHz}$, these equations must be valid for $t \leq 2$ microseconds and $T \leq 3$ microseconds respectively for the rectangular and half cosine inputs. It was found from signals simulated by these input functions that these equations hold good as long as these conditions are met.

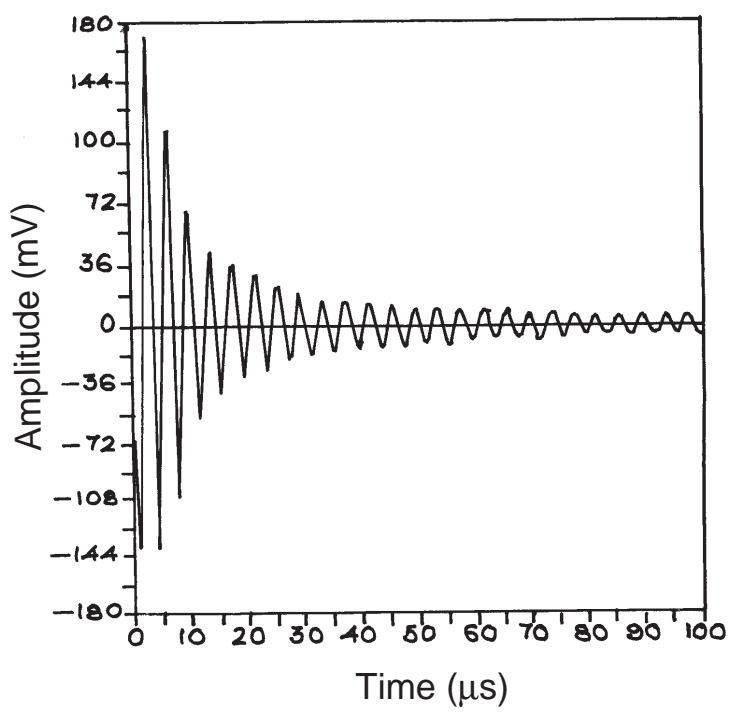

Figure 14. Signal due to half cosine source $T=5$ microseconds and $A=3$ microbar. 


\section{Significance of peak amplitude and rise time}

The relation that pulse width is twice the rise time and the equations for peak amplitude, (20), shows that rise time and peak amplitude contain complete information about triangular source parameters. This is obvious from (20) which can be written, by rearranging and substituting $R_{t}=T / 2$, as

$$
A=P a /\left[C T \int_{f 1}^{f 2} \frac{\sin ^{2}\left(\Pi f R_{t}\right)}{\left(\Pi f R_{t}\right)^{2}} \mathrm{~d} f\right]
$$

This is true about Gaussian, rectangular (under the condition $f_{2} \leq 1 / T$ ), and half cosine (under the condition $f_{2} \leq 3 / 2 T$ ) inputs. In brief, we can conclude from the foregoing that peak amplitude and the rise time are significant parameters for source characterization.

\section{Conclusion}

Theoretical expressions for peak amplitude and rise time of acoustic emission signals are derived. Correctness of these expressions are established using simulated signals. Inferences drawn from these expressions justify the use of these parameters for acoustic emission source characterization.

We thank Prof. Thomas Chacko of the languages section for going through the paper.

\section{Appendix A}

From (16) we get

$$
\begin{aligned}
g_{T}(t) & \leq C A T \int_{f 1}^{f 2}\left|\frac{\sin ^{2}(\Pi f T / 2)}{(\Pi f T / 2)^{2}} \cos 2 \Pi f\left[t-\left(t_{0}+T / 2\right)\right]\right| \mathrm{d} f \\
& \left.\leq C A T \int_{f 1}^{f 2} \frac{\sin ^{2}(\Pi f T / 2)}{(\Pi f T / 2)^{2}} \mid \cos 2 \Pi f\left[t-t_{0}+T / 2\right)\right] \mid \mathrm{d} f \\
& \leq C A T \int_{f 1}^{f 2} \frac{\sin ^{2}(\Pi f T / 2)}{(\Pi f T / 2)^{2}} \mathrm{~d} f \\
\frac{\mathrm{d} g_{t}(t)}{\mathrm{d} t} & =C A T \int_{f 1}^{f 2} \frac{\sin ^{2}(\Pi f T / 2)}{(\Pi f T / 2)^{2}}(\mathrm{~d} / \mathrm{d} t)\left\{\cos 2 \Pi f\left[t-\left(t_{0}+T / 2\right)\right]\right\} \mathrm{d} f,
\end{aligned}
$$

that is,

$$
\begin{gathered}
\frac{\mathrm{d} g_{T}(t)}{\mathrm{d} t}=-2 C A T \int_{f 1}^{f 2} \frac{\sin ^{2}(\Pi f T / 2)}{(\Pi f T / 2)^{2}} \sin 2 \Pi f\left[t-\left(t_{0}+T / 2\right)\right] \Pi f \mathrm{~d} f, \\
\frac{\mathrm{d}^{2} g_{T}(t)}{\mathrm{d} t^{2}}=-4 C A T \int_{f 1}^{f 2} \frac{\sin ^{2}(\Pi f T / 2)}{(\Pi f T / 2)^{2}} \cos 2 \Pi f\left[t-\left(t_{0}+T / 2\right)\right](\Pi f) \mathrm{d} f,
\end{gathered}
$$




$$
\frac{\mathrm{d} g_{T}(t)}{\mathrm{d} t}=0 \text { when } t=t_{0}+T / 2
$$

and

$$
\frac{\mathrm{d}^{2} g_{T}(t)}{\mathrm{d} t}<0 \text { at } t=t_{0}+T / 2 .
$$

Hence $g_{T}(t)$ has a maximum at $t=t_{0}+T / 2$ which is given by

$$
g T\left(t_{0}+T / 2\right)=C A T \int_{f 1}^{f 2} \frac{\sin ^{2}(\Pi f T / 2)}{(\Pi f T / 2)^{2}} \mathrm{~d} f .
$$

By comparing (A2) with (A1) we find that $g_{T}(t)$ attains its upper bound, given by (A1), at $t=t_{0}+T / 2$ thus completing the proof.

Assume that we have a $t=t^{\prime} \neq t_{0}+T / 2$ such that $g_{T}\left(t^{\prime}\right)=\max g_{T}(t)=P_{a}$. This implies that $\cos 2 \Pi f\left[t^{\prime}-\left(t_{0}+T / 2\right)\right]=1$, for all $f_{1} \leq f \leq f_{2}$ (otherwise $g_{T}\left(t^{\prime}\right)<$ $\left.C A T \int_{f 1}^{f 2}\left\{\left[\sin ^{2}(\Pi f T / 2)\right] /(\Pi f T / 2)^{2}\right\} \mathrm{d} f=P_{a}\right)$ implying that $2 \Pi f\left[t-\left(t_{0}+T / 2\right)\right]=0$ for all $f_{1} \leq f \leq f_{2}$. This is impossible and hence, our assumption is wrong and $P_{a}$ occurs uniquely at $t=t_{0}+T / 2$.

We have seen that $P_{a}$ occurs at $t=t_{0}+T / 2$. Suppose we treat the instant when the signal first appears at the transducer output as the time origin. This is equivalent to treating the instant when the transient pulse is applied to the transducer as the time origin with $t_{0}=0$. Under this condition we get

$$
R_{t}=T / 2 .
$$

\section{References}

Bhat M R, Murthy C R L 1993 Fatigue damage stages in unidirectional glass-fibre-epoxy composites: Identification through acoustic emission technique. Int. J. Fatigue 15: 401-405

Hill R, Stephens R W B 1974 Simple theory of acoustic emission-a consideration of measurement parameters. Acoustica 31: 225-230

Krylov V I 1966 Approximate calculation of integrals (NewYork: MacMillan)

Murthy C R L, Majeed M A, Pathak S C, Rao A K 1985 In-Flight monitoring for incipient cracks in an aero engine mount: An approach through pattern recognition. J. Acoust. Emission 4: S147-S150

Murthy C R L, Dattaguru B, Rao A K 1987 Application of pattern recognition concepts to acoustic emission signal analysis. J. Acoust. Emission : 19-28

Papoulis A 1977 Signal analysis (New Delhi:McGraw-Hill)

Salivahanan S, Vallavaraj A, Gnanapriya C 2000 Digital signal processing (New Delhi: Tata McGrawHill)

Stephens R W B, Pollock A A, 1971 Waveforms and frequency spectra of acoustic emission. J. Acoust Soc. Am. 50: 904-910

Stroud A H, Secrest D 1966, Gaussian quadrature formulas (Englewood Cliffs, NJ: Prentice-Hall) 